\title{
ON JORDANIAN $\mathrm{U}_{h, \alpha}(\mathrm{gl}(2))$ ALGEBRA AND ITS $T$ MATRICES VIA A CONTRACTION METHOD
}

\author{
R. CHAKRABARTI ${ }^{1}$ and C. QUESNE ${ }^{2, *}$ \\ ${ }^{1}$ Department of Theoretical Physics, University of Madras, \\ Guindy Campus, Madras-600025, India \\ 2 Physique Nucléaire Théorique et Physique Mathématique, \\ Université Libre de Bruxelles, Campus de la Plaine CP229, \\ Boulevard du Triomphe, B-1050 Brussels, Belgium
}

\begin{abstract}
The $R_{h}^{j_{1} ; j_{2}}$ matrices of the Jordanian $\mathrm{U}_{h}(\mathrm{sl}(2))$ algebra at arbitrary dimensions may be obtained from the corresponding $R_{q}^{j_{1} ; j_{2}}$ matrices of the standard $q$-deformed $\mathrm{U}_{q}(\mathrm{sl}(2))$ algebra through a contraction technique. By extending this method, the coloured two-parametric $(h, \alpha)$ Jordanian $R_{h, \alpha}^{j_{1}, z_{1} ; j_{2}, z_{2}}$ matrices of the $\mathrm{U}_{h, \alpha}(\mathrm{gl}(2))$ algebra may be derived from the corresponding coloured $R_{q, \lambda}^{j_{1}, z_{1} ; j_{2}, z_{2}}$ matrices of the standard $(q, \lambda)$-deformed $\mathrm{U}_{q, \lambda}(\mathrm{gl}(2))$ algebra. Moreover, by using the contraction process as a tool, the coloured $T_{h, \alpha}^{j, z}$ matrices for arbitrary $(j, z)$ representations of the Jordanian $\operatorname{Fun}_{h, \alpha}(\mathrm{GL}(2))$ algebra may be extracted from the corresponding $T_{q, \lambda}^{j, z}$ matrices of the standard $\operatorname{Fun}_{q, \lambda}(\mathrm{GL}(2))$ algebra.
\end{abstract}

*Directeur de recherches FNRS; E-mail address: cquesne@ulb.ac.be 


\section{Introduction}

The Lie group GL(2) only admits (up to isomorphism) two distinct quantum group deformations with central quantum determinant [1]: the standard $q$-deformation $\operatorname{Fun}_{q}(\mathrm{GL}(2))$ [2], and the Jordanian deformation $\operatorname{Fun}_{h}(\mathrm{GL}(2))$ [3]. On the quantum algebra level, the Jordanian deformation $\mathrm{U}_{h}(\mathrm{sl}(2))$ of the classical enveloping algebra $\mathrm{U}(\operatorname{sl}(2))$ was first considered in Ref. [4], and its universal $\mathcal{R}_{h}$ matrix was given in Ref. [5]. The fundamental representation of $\mathrm{U}_{h}(\mathrm{sl}(2))$, which remains undeformed, was obtained in Ref. [由], while the other finite-dimensional highest-weight representations were first studied in Ref. [6].

Both $\operatorname{Fun}_{q}(\mathrm{GL}(2))$ and $\operatorname{Fun}_{h}(\mathrm{GL}(2))$ quantum groups may be viewed as special cases of two-parametric deformations, $\operatorname{Fun}_{q, \lambda}(\mathrm{GL}(2))$ [7] and $\operatorname{Fun}_{h, \alpha}(\mathrm{GL}(2))$ [8], respectively. The quantum algebra $\mathrm{U}_{h, \alpha}(\mathrm{gl}(2))$, dual to the two-parametric Jordanian quantum group $\operatorname{Fun}_{h, \alpha}(\mathrm{GL}(2))$, was found in Ref. [9]. It was shown there that with an appropriate choice of parameters, the algebraic properties of the two-parametric Jordanian $\mathrm{U}_{h, \alpha}(\operatorname{gl}(2))$ algebra coincide with those of the single-parametric $\mathrm{U}_{h}(\operatorname{gl}(2))$ one. The second parameter $\alpha$, however, plays a manifest role in the coalgebraic properties. An independent construction of the Hopf algebra $\mathrm{U}_{h, \alpha}(\mathrm{gl}(2))$ was carried out in Ref. [10], together with a determination of the corresponding universal $\mathcal{R}_{h, \alpha}$ matrix.

Some related coloured Hopf algebraic structures were also introduced. In Ref. [11], the single-parametric Jordanian $\mathrm{U}_{h}(\mathrm{gl}(2))$ algebra was extended to a coloured Hopf algebra $\mathrm{U}^{c}(\mathrm{gl}(2))$, endowed with a coloured universal $\mathcal{R}^{c}$ matrix. More recently, a coloured two-parametric Jordanian $R_{h, \alpha}^{z_{1} ; z_{2}}$ matrix, corresponding to the fundamental representation, was constructed, and used to build a coloured quantum group $\operatorname{Fun}_{h, \alpha}^{z_{1} ; z_{2}}(\mathrm{GL}(2))$ [10], thereby generalizing to the Jordanian context a previous work in the standard $q$-deformed context [12].

Two useful tools have been devised for studying Jordanian deformations. One of them is a contraction procedure that allows one to construct the latter from standard deformations [13]: a similarity transformation of the defining $R_{q}$ and $T_{q}$ (resp. $R_{q, \lambda}$ 
and $\left.T_{q, \lambda}\right)$ matrices of $\operatorname{Fun}_{q}(\mathrm{SL}(2))$ (resp. $\left.\operatorname{Fun}_{q, \lambda}(\mathrm{GL}(2))\right)$ is performed using a matrix singular itself in the $q \rightarrow 1$ limit, but in such a way that the transformed matrices are non-singular, and yield the defining $R_{h}$ and $T_{h}$ (resp. $R_{h, \alpha}$ and $T_{h, \alpha}$ ) matrices of $\operatorname{Fun}_{h}(\mathrm{SL}(2))$ (resp. Fun $\left.{ }_{h, \alpha}(\mathrm{GL}(2))\right)$. Such a contraction technique can be generalized to higher-dimensional quantum groups 14].

The other tool is a nonlinear invertible map between the generators of $\mathrm{U}_{h}(\operatorname{sl}(2))$ and $\mathrm{U}(\mathrm{sl}(2))$ [15]. This map yields an explicit and simple method for constructing the finite-dimensional irreducible representations of $\mathrm{U}_{h}(\operatorname{sl}(2))$ [15], and for studying various related problems [16, 17.

Recently, an operational generalization of the contraction method combining both tools was proposed 18]. It yields the Jordanian deformation of $\mathrm{U}(\mathrm{sl}(N))$ from the standard one along with a nonlinear map from the $h$-Borel subalgebra on the corresponding classical Borel subalgebra, which in the case of $\mathrm{U}_{h}(\mathrm{sl}(2))$ can be extended to the whole algebra. By using such a generalization, the $R_{h}^{\frac{1}{2} ; j}$ matrix of the $\left(\frac{1}{2} \otimes j\right)$ representation of $\mathrm{U}_{h}(\mathrm{sl}(2))$ was obtained from the corresponding $R_{q}^{\frac{1}{2} ; j}$ matrix of the standard $q$-deformed $\mathrm{U}_{q}(\operatorname{sl}(2))$ algebra [18]. Furthermore, it was shown that the Drinfeld twist operator [19], relating the usual classical U(sl(2)) coalgebraic properties with the nontrivial coalgebraic structure of the Jordanian $\mathrm{U}_{h}(\operatorname{sl}(2))$ algebra, can be found as a series expansion in the deformation parameter $h$ [18].

The purpose of the present paper is threefold. Firstly, in Sec. 2, we wish to complete the work of Ref. [18] by providing higher order terms in the expansion of the Drinfeld twist operator, and by demonstrating that the $h$-Jordanian $R_{h}^{j_{1} ; j_{2}}$ matrices of arbitrary $\left(j_{1} \otimes j_{2}\right)$ representations of $\mathrm{U}_{h}(\mathrm{sl}(2))$ may be obtained by contracting the corresponding standard $R_{q}^{j_{1} ; j_{2}}$ matrices.

Secondly, in Sec. 3, we wish to extend Ref. [18] in two ways: by going from the single-parametric case to the two-parametric one, and from $\left(j_{1} \otimes j_{2}\right)$ standard representations to $\left(j_{1}, z_{1} \otimes j_{2}, z_{2}\right)$ coloured representations, where the colour parameter $z$ assumes arbitrary distinct values in different sectors of the tensor product space. We will prove that the contraction scheme provides a mechanism for extracting 
the $R_{h, \alpha}^{j_{1}, z_{1} ; j_{2}, z_{2}}$ matrix in an arbitrary $\left(j_{1}, z_{1} \otimes j_{2}, z_{2}\right)$ coloured representation of the $\mathrm{U}_{h, \alpha}(\mathrm{gl}(2))$ algebra from the corresponding $R_{q, \lambda}^{j_{1}, z_{1} ; j_{2}, z_{2}}$ matrix of the two-parametric standard $\mathrm{U}_{q, \lambda}(\mathrm{gl}(2))$ algebra. As a result, we will get solutions of the coloured YangBaxter equation

$$
R_{12}^{j_{1}, z_{1} ; j_{2}, z_{2}} R_{13}^{j_{1}, z_{1} ; j_{3}, z_{3}} R_{23}^{j_{2}, z_{2} ; j_{3}, z_{3}}=R_{23}^{j_{2}, z_{2} ; j_{3}, z_{3}} R_{13}^{j_{1}, z_{1} ; j_{3}, z_{3}} R_{12}^{j_{1}, z_{1} ; j_{2}, z_{2}}
$$

where we have suppressed the subscripts $(h, \alpha)$.

Thirdly, in Sec. 4, we wish to apply the contraction process of Ref. [18 to the construction of an entirely new type of Jordanian deformed objects, namely the finite-dimensional coloured representations of $(h, \alpha)$-deformed group-like elements $T_{h, \alpha}^{j, z}$ of $\operatorname{Fun}_{h, \alpha}(\mathrm{GL}(2))$. In this respect, it should be stressed that if the universal $\mathcal{T}_{q, \lambda}$ matrix, acting as a dual form between the $(q, \lambda)$-deformed standard Hopf algebras $\operatorname{Fun}_{q, \lambda}(\mathrm{GL}(2))$ and $\mathrm{U}_{q, \lambda}(\mathrm{gl}(2))$, is well known [20], as well as its finite-dimensional coloured representations $T_{q, \lambda}^{j, z}$ [21], nothing similar is available yet for Jordanian Hopf algebras (but for the defining $T_{h, \alpha}$ matrix). Here, we will prove that the $T_{q, \lambda}^{j, z}$ matrices generate, through the contraction procedure, the corresponding Jordanian $T_{h, \alpha}^{j, z}$ matrices. In this way, we will construct solutions of the coloured inverse scattering equation

$$
R_{h, \alpha}^{j_{1}, z_{1} ; j_{2}, z_{2}}\left(T_{h, \alpha}^{j_{1}, z_{1}} \otimes 1\right)\left(1 \otimes T_{h, \alpha}^{j_{2}, z_{2}}\right)=\left(1 \otimes T_{h, \alpha}^{j_{2}, z_{2}}\right)\left(T_{h, \alpha}^{j_{1}, z_{1}} \otimes 1\right) R_{h, \alpha}^{j_{1}, z_{1} ; j_{2}, z_{2}} .
$$

\section{The $R_{h}$ Matrices of the $\mathrm{U}_{h}(\mathrm{sl}(2))$ Algebra as Contraction Limits}

We start by enlisting the Hopf structure of the single-parametric Jordanian algebra $\mathrm{U}_{h}(\mathrm{sl}(2))$ 四. The algebra reads

$$
[H, X]=2 \frac{\sinh h X}{h}, \quad[H, Y]=-Y(\cosh h X)-(\cosh h X) Y, \quad[X, Y]=H .
$$

The non-cocommutative coproduct, counit and the antipode maps assume the form

$$
\Delta_{h}(X)=X \otimes 1+1 \otimes X, \quad \Delta_{h}(Y)=Y \otimes T+T^{-1} \otimes Y,
$$




$$
\begin{aligned}
\Delta_{h}(H) & =H \otimes T+T^{-1} \otimes H, \\
\epsilon_{h}(\mathcal{X}) & =0 \quad \forall \mathcal{X} \in\{X, Y, H\}, \\
S_{h}(X) & =-X, \quad S_{h}(Y)=-T Y T^{-1}, \quad S_{h}(H)=-T H T^{-1},
\end{aligned}
$$

where $T=\exp (h X)$. The universal $\mathcal{R}_{h}$ matrix of the triangular Hopf algebra $\mathrm{U}_{h}(\mathrm{sl}(2))$ is given [5] in a convenient form by

$$
\mathcal{R}_{h}=\exp (-h X \otimes T H) \exp (h T H \otimes X) .
$$

For a quasitriangular Hopf algebra $U$, the universal $\mathcal{R}$ matrix, an invertible element in $U \otimes U$, satisfies the relations

$$
\begin{aligned}
(\Delta \otimes \mathrm{id}) \mathcal{R} & =\mathcal{R}_{13} \mathcal{R}_{23}, \quad(\mathrm{id} \otimes \mathcal{R})=\mathcal{R}_{13} \mathcal{R}_{12}, \\
\sigma \circ \Delta(\mathcal{X}) & =\mathcal{R} \Delta(\mathcal{X}) \mathcal{R}^{-1} \quad \forall \mathcal{X} \in U,
\end{aligned}
$$

where $\sigma$ is the permutation in $U \otimes U$. For a triangular Hopf algebra, the additional property $\mathcal{R}^{-1}=\mathcal{R}_{21}$ holds.

An invertible nonlinear map of the generating elements of the $\mathrm{U}_{h}(\mathrm{sl}(2))$ algebra on the elements of the classical $\mathrm{U}(\mathrm{sl}(2))$ algebra plays [18] a pivotal role in obtaining the $h$-Jordanian $R_{h}^{\frac{1}{2} ; j}$ matrix in the $\left(\frac{1}{2} \otimes j\right)$ representation as a suitable contraction limit of the corresponding standard $R_{q}^{\frac{1}{2} ; j}$ matrix. The map reads [18

$$
T=\tilde{T}, \quad Y=J_{-}-\frac{1}{4} h^{2} J_{+}\left(J_{0}^{2}-1\right), \quad H=\left(1+\left(h J_{+}\right)^{2}\right)^{1 / 2} J_{0},
$$

where $\tilde{T}=h J_{+}+\left(1+\left(h J_{+}\right)^{2}\right)^{1 / 2}$. The elements $\left(J_{ \pm}, J_{0}\right)$ are the generators of the classical $\mathrm{U}(\mathrm{sl}(2))$ algebra:

$$
\begin{aligned}
& {\left[J_{0}, J_{ \pm}\right]= \pm 2 J_{ \pm}, \quad\left[J_{+}, J_{-}\right]=J_{0},} \\
& \Delta_{0}\left(J_{\zeta}\right)=J_{\zeta} \otimes 1+1 \otimes J_{\zeta}, \quad \epsilon_{0}\left(J_{\zeta}\right)=0, \quad S_{0}\left(J_{\zeta}\right)=-J_{\zeta} \quad \forall \zeta \in\{ \pm, 0\} .
\end{aligned}
$$

Following Drinfeld's arguments [19], it is possible to construct a twist operator $G \in \mathrm{U}(\mathrm{sl}(2))^{\otimes 2}[[h]]$ relating the $h$-Jordanian coalgebraic structure (2.2) with the 
corresponding classical structure (2.7). For an invertible map $m:(X, Y, H) \rightarrow$ $\left(J_{ \pm}, J_{0}\right), m^{-1}:\left(J_{ \pm}, J_{0}\right) \rightarrow(X, Y, H)$, the following relations hold:

$$
\begin{aligned}
(m \otimes m) \circ \Delta_{h} \circ m^{-1}(\mathcal{X}) & =G \Delta_{0}(\mathcal{X}) G^{-1}, \\
m \circ S_{h} \circ m^{-1}(\mathcal{X}) & =g S_{0}(\mathcal{X}) g^{-1},
\end{aligned}
$$

where $\mathcal{X} \in \mathrm{U}(\operatorname{sl}(2))[[h]]$. The transforming operator $g(\in \mathrm{U}(\mathrm{sl}(2))[[h]])$, and its inverse may be expressed as

$$
g=\mu \circ\left(\mathrm{id} \otimes S_{0}\right) G, \quad g^{-1}=\mu \circ\left(S_{0} \otimes \mathrm{id}\right) G^{-1},
$$

where $\mu$ is the multiplication map.

For the map (2.5), we have the following construction:

$$
\begin{aligned}
G= & 1 \otimes 1-\frac{1}{2} h r+\frac{1}{8} h^{2}\left[r^{2}+2\left(J_{+} \otimes J_{+}\right) \Delta_{0}\left(J_{0}\right)\right] \\
& -\frac{1}{48} h^{3}\left[r^{3}+6\left(J_{+} \otimes J_{+}\right) \Delta_{0}\left(J_{0}\right) r-4\left(\Delta_{0}\left(J_{+}\right)\right)^{2} r\right] \\
& +\frac{1}{384} h^{4}\left[r^{4}-16\left(\Delta_{0}\left(J_{+}\right)\right)^{2} r^{2}+12\left(J_{+} \otimes J_{+}\right) \Delta_{0}\left(J_{0}\right) r^{2}\right. \\
& +12\left(\left(J_{+} \otimes J_{+}\right) \Delta_{0}\left(J_{0}\right)\right)^{2}+6\left(J_{+}^{2} \otimes 1-1 \otimes J_{+}^{2}\right)^{2} \Delta_{0}\left(J_{0}\right) \\
& +12\left(\Delta_{0}\left(J_{+}\right)\right)^{2}\left(J_{+}^{2} \otimes 1+1 \otimes J_{+}^{2}\right) \Delta_{0}\left(J_{0}\right) \\
& \left.-8 \Delta_{0}\left(J_{+}\right)\left(J_{+}^{3} \otimes 1+1 \otimes J_{+}^{3}\right) \Delta_{0}\left(J_{0}\right)-10\left(\Delta_{0}\left(J_{+}\right)\right)^{4} \Delta_{0}\left(J_{0}\right)\right] \\
& +O\left(h^{5}\right), \\
g= & 1+h J_{+}\left(1+h^{2} J_{+}^{2}\right)^{1 / 2}+h^{2} J_{+}^{2}, \\
g^{-1}= & 1-h J_{+}\left(1+h^{2} J_{+}^{2}\right)^{-1 / 2},
\end{aligned}
$$

where the Jordanian classical $r$ matrix reads $r=J_{0} \otimes J_{+}-J_{+} \otimes J_{0}$. The transforming operator $g$ is obtained in a closed form in (2.10). The first non-trivial term $O\left(h^{2}\right)$ in the series expansion of $G$ in (2.10) was previously evaluated [18]. The series expansion of the twist operator $G$ may be developed up to an arbitrary order in $h$. It may be verified that the expansion (2.10), in powers of $h$, of the twist operator $G$ corresponding to the map (2.5) satisfies the cocycle condition

$$
(1 \otimes G)\left(\mathrm{id} \otimes \Delta_{0}\right) G=(G \otimes 1)\left(\Delta_{0} \otimes \mathrm{id}\right) G
$$


up to the desired order. The universal $\mathcal{R}_{h}$ matrix (2.3), used in conjunction with the map (2.5), may be recast in the form

$$
\mathcal{R}_{h}=(\sigma \circ G) G^{-1}
$$

valid up to an arbitrary order in the expansion (2.10).

In Ref. [18], the $R_{q}^{\frac{1}{2} ; j}$ matrix of the standard $q$-deformed $\mathrm{U}_{q}(\operatorname{sl}(2))$ algebra has been found to yield, after a suitable transformation and a subsequent $q \rightarrow 1$ limiting process, the corresponding $h$-Jordanian $R_{h}^{\frac{1}{2} ; j}$ matrix. A well-defined construction along this route for the $R_{h}^{\frac{1}{2} ; j}$ matrix exists as the singularities systematically cancel. Similar construction of $R_{h}^{j_{1} ; j_{2}}$ matrices may be continued for arbitrary $\left(j_{1} \otimes j_{2}\right)$ representations.

To this end, we proceed with the universal $\mathcal{R}_{q}$ matrix of the $\mathrm{U}_{q}(\operatorname{sl}(2))$ algebra, given by [22]

$$
\mathcal{R}_{q}=q^{\frac{1}{2} \hat{J}_{0} \otimes \hat{J}_{0}} \exp _{q^{-2}}\left(\left(1-q^{-2}\right) q^{\hat{J}_{0} / 2} \hat{J}_{+} \otimes q^{-\hat{J}_{0} / 2} \hat{J}_{-}\right)
$$

where $\exp _{q}(\mathcal{X})=\sum_{n=0}^{\infty} \mathcal{X}^{n} /\{n\}_{q} !,\{n\}_{q} !=\{n\}_{q}\{n-1\}_{q} \cdots\{1\}_{q}$ if $n \in \mathbb{N}^{+},\{0\}_{q} !=1$, and $\{n\}_{q}=\left(1-q^{n}\right) /(1-q)$. The generators of the standard $\mathrm{U}_{q}(\operatorname{sl}(2))$ algebra satisfy the commutation relations

$$
q^{\hat{J}_{0}} \hat{J}_{ \pm} q^{-\hat{J}_{0}}=q^{ \pm 2} \hat{J}_{ \pm}, \quad\left[\hat{J}_{+}, \hat{J}_{-}\right]=\left[\hat{J}_{0}\right]_{q}
$$

where $[\mathcal{X}]_{q}=\left(q^{\mathcal{X}}-q^{\mathcal{X}}\right) /\left(q-q^{-1}\right)$.

Following Ref. [18], we introduce a transforming matrix $M$, singular in the $q \rightarrow 1$ limit, as

$$
M=\mathrm{E}_{q}\left(\eta \hat{J}_{+}\right)
$$

where $\mathrm{E}_{q}(\mathcal{X})=\sum_{n=0}^{\infty} \mathcal{X}^{n} /[n]_{q} !,[n]_{q} !=[n]_{q}[n-1]_{q} \cdots[1]_{q}$ if $n \in \mathbb{N}^{+},[0]_{q} !=1$, and $\eta=h /(q-1)$. The standard $R_{q}^{j_{1} ; j_{2}}$ matrix may now be subjected to a similarity transformation followed by a $q \rightarrow 1$ limiting process:

$$
\tilde{R}_{h}^{j_{1} ; j_{2}}=\lim _{q \rightarrow 1}\left[\left(M_{j_{1}}^{-1} \otimes M_{j_{2}}^{-1}\right) R_{q}^{j_{1} ; j_{2}}\left(M_{j_{1}} \otimes M_{j_{2}}\right)\right]
$$


The matrix $\tilde{R}_{h}^{j_{1} ; j_{2}}$ obtained by the above prescription is non-singular, and may be seen to coincide, upon application of nonlinear map (2.5), with the corresponding $R_{h}^{j_{1} ; j_{2}}$ obtained directly from the expression (2.3) of the universal $\mathcal{R}_{h}$ matrix.

The fundamental $j=\frac{1}{2}$ representation of the $\mathrm{U}_{q}(\mathrm{sl}(2))$ algebra remains undeformed, and the corresponding $\tilde{R}_{h}^{\frac{1}{2} ; j}$ matrix in (2.16) reads 18

$$
\tilde{R}_{h}^{\frac{1}{2} ; j}=\left(\begin{array}{cc}
\tilde{T} & -\frac{1}{2} h\left(\tilde{T}+\tilde{T}^{-1}\right) \\
0 & J_{0}+\frac{1}{2} h\left(\tilde{T}-\tilde{T}^{-1}\right) \\
0 & \tilde{T}^{-1}
\end{array}\right) .
$$

Using similar techniques, the higher-dimensional $R_{h}$ matrices may also be studied. The general features of the construction (2.16) may be observed in analysing the results for $(1 \otimes j)$, and $\left(\frac{3}{2} \otimes j\right)$ representations, respectively. For convenience, we choose the $(2 j+1)$-dimensional irreducible representation of the $\mathrm{U}_{q}(\operatorname{sl}(2))$ algebra as follows:

$$
\begin{aligned}
\hat{J}_{+}|j m\rangle & =[j-m]_{q}[j+m+1]_{q}|j m+1\rangle, \quad \hat{J}_{-}|j m\rangle=|j m-1\rangle, \\
\hat{J}_{0}|j m\rangle & =m|j m\rangle,
\end{aligned}
$$

where $m=j, j-1, \ldots,-(j-1),-j$. The transforming matrix $M$ in (2.15) may now be obtained for $j=1$, and $j=\frac{3}{2}$ representations as

$$
M_{j=1}=\left(\begin{array}{ccc}
1 & {[2]_{q} \eta} & {[2]_{q} \eta^{2}} \\
0 & 1 & {[2]_{q} \eta} \\
0 & 0 & 1
\end{array}\right), \quad M_{j=\frac{3}{2}}=\left(\begin{array}{cccc}
1 & {[3]_{q} \eta} & {[3]_{q} ! \eta^{2}} & {[3]_{q} ! \eta^{3}} \\
0 & 1 & {[2]_{q}^{2} \eta} & {[3]_{q} ! \eta^{2}} \\
0 & 0 & 1 & {[3]_{q} \eta} \\
0 & 0 & 0 & 1
\end{array}\right) .
$$

Following the recipe (2.16), the $\tilde{R}_{h}^{1 ; j}$ and $\tilde{R}_{h}^{\frac{3}{2} ; j}$ matrices may be realized as

$$
\tilde{R}_{h}^{1 ; j}=\left(\begin{array}{ccc}
\tilde{T}^{2} & -h\left(\tilde{T}^{2}+1\right) J_{0} & \frac{1}{2} h^{2}\left[\left(\tilde{T}+\tilde{T}^{-1}\right)^{2} J_{0}^{2}-4\left(\tilde{T}^{2}-\tilde{T}^{-2}\right)\right. \\
& & \left.+4\left(\tilde{T}^{-2}-1\right) J_{0}\right] \\
0 & 1 & -h\left[\left(\tilde{T}^{-2}+1\right) J_{0}+2\left(\tilde{T}^{-2}-1\right)\right] \\
0 & 0 & \tilde{T}^{-2}
\end{array}\right),
$$


and

$$
\tilde{R}_{h}^{\frac{3}{2} ; j}=\left(\begin{array}{cccc}
\tilde{T}^{3} & \tilde{A} & \tilde{B} & \tilde{C} \\
0 & \tilde{T} & \tilde{D} & \tilde{E} \\
0 & 0 & \tilde{T}^{-1} & \tilde{F} \\
0 & 0 & 0 & \tilde{T}^{-3}
\end{array}\right)
$$

where

$$
\begin{aligned}
\tilde{A}= & -\frac{3}{2} h\left[\tilde{T}^{3}\left(J_{0}+1\right)+\tilde{T}\left(J_{0}-1\right)\right], \\
\tilde{B}= & \frac{3}{2} h^{2}\left[\tilde{T}^{3}\left(J_{0}-1\right)\left(J_{0}+3\right)+2 \tilde{T}\left(J_{0}-1\right)^{2}+\tilde{T}^{-1}\left(J_{0}+1\right)^{2}\right], \\
\tilde{C}= & -\frac{3}{4} h^{3}\left[\tilde{T}^{3}\left(J_{0}-3\right)\left(J_{0}+1\right)\left(J_{0}+5\right)+3 \tilde{T}\left(J_{0}-3\right)\left(J_{0}-1\right)\left(J_{0}+1\right)\right. \\
& \left.+3 \tilde{T}^{-1}\left(J_{0}-3\right)\left(J_{0}+1\right)^{2}+\tilde{T}^{-3}\left(J_{0}+1\right)\left(J_{0}+3\right)\left(J_{0}+5\right)\right], \\
\tilde{D}= & -2 h\left[\tilde{T}\left(J_{0}-1\right)+\tilde{T}^{-1}\left(J_{0}+1\right)\right], \\
\tilde{E}= & \frac{3}{2} h^{2}\left[\left(\tilde{T}+2 \tilde{T}^{-1}\right)\left(J_{0}-3\right)\left(J_{0}+1\right)+\tilde{T}^{-3}\left(J_{0}+3\right)^{2}\right], \\
\tilde{F}= & -\frac{3}{2} h\left[\tilde{T}^{-1}\left(J_{0}-3\right)+\tilde{T}^{-3}\left(J_{0}+3\right)\right] .
\end{aligned}
$$

In the above computational procedure of the $\tilde{R}_{h}^{j_{1} ; j_{2}}$ matrices, obtained à la (2.16), the order of the singularity in the $q \rightarrow 1$ limit increases by one for each step away from the diagonal, the rightmost corner element being the most singular. The singularities in the said $q \rightarrow 1$ limit, however, all cancel yielding finite results as in $(2.20)$, and (2.21).

To prove the equivalence of the $\tilde{R}_{h}^{j_{1} ; j_{2}}$ matrices obtained above as per our contraction procedure, and the corresponding $R_{h}^{j_{1} ; j_{2}}$ read directly from the universal $\mathcal{R}_{h}$ matrix (2.3), we first use the map (2.5) to obtain a representation of the $h$-Jordanian algebra (2.1). A $(2 j+1)$-dimensional representation of the classical algebra (2.6)

$$
\begin{aligned}
J_{+}|j m\rangle & =(j-m)(j+m+1)|j m+1\rangle, \quad J_{-}|j m\rangle=|j m-1\rangle, \\
J_{0}|j m\rangle & =m|j m\rangle,
\end{aligned}
$$

now, via the nonlinear map (2.5), immediately furnishes the corresponding $(2 j+1)$ dimensional representation of the $h$-Jordanian algebra (2.1). For the $j=\frac{1}{2}$ case, 
the generators remain undeformed. For the $j=1$ and $j=\frac{3}{2}$ cases, we list the representations of $\mathrm{U}_{h}(\mathrm{sl}(2))$ generators below:

$$
\begin{aligned}
& (j=1) \\
& X=\left(\begin{array}{lll}
0 & 2 & 0 \\
0 & 0 & 2 \\
0 & 0 & 0
\end{array}\right), \quad Y=\left(\begin{array}{ccc}
0 & \frac{1}{2} h^{2} & 0 \\
1 & 0 & -\frac{3}{2} h^{2} \\
0 & 1 & 0
\end{array}\right), \\
& H=\left(\begin{array}{ccc}
2 & 0 & -4 h^{2} \\
0 & 0 & 0 \\
0 & 0 & -2
\end{array}\right) \\
& \left(j=\frac{3}{2}\right) \\
& X=\left(\begin{array}{cccc}
0 & 3 & 0 & -6 h^{2} \\
0 & 0 & 4 & 0 \\
0 & 0 & 0 & 3 \\
0 & 0 & 0 & 0
\end{array}\right), \quad Y=\left(\begin{array}{cccc}
0 & 0 & 0 & 0 \\
1 & 0 & 0 & 0 \\
0 & 1 & 0 & -6 h^{2} \\
0 & 0 & 1 & 0
\end{array}\right), \\
& H=\left(\begin{array}{cccc}
3 & 0 & -6 h^{2} & 0 \\
0 & 1 & 0 & -18 h^{2} \\
0 & 0 & -1 & 0 \\
0 & 0 & 0 & -3
\end{array}\right)
\end{aligned}
$$

Using the above representations in the expression (2.3) of the universal $\mathcal{R}_{h}$ matrix, we obtain

$$
\begin{gathered}
R_{h}^{\frac{1}{2} ; j}=\left(\begin{array}{cc}
T & -h H+\frac{1}{2} h\left(T-T^{-1}\right) \\
0 & T^{-1}
\end{array}\right) \\
R_{h}^{1 ; j}=\left(\begin{array}{ccc}
T^{2} & -2 h T H & -2 h^{2}\left[T^{2}-T^{-2}+2 T H\left(T^{-2}-1\right)-(T H)^{2} T^{-2}\right] \\
0 & 1 & -2 h\left(T H T^{-2}-T^{-2}+1\right) \\
0 & 0 & T^{-2}
\end{array}\right),
\end{gathered}
$$

and

$$
R_{h}^{\frac{3}{2} ; j}=\left(\begin{array}{cccc}
T^{3} & A & B & C \\
0 & T & D & E \\
0 & 0 & T^{-1} & F \\
0 & 0 & 0 & T^{-3}
\end{array}\right)
$$


where

$$
\begin{aligned}
A= & \frac{3}{2} h\left(T^{2}-1-2 T H\right) T, \\
B= & -\frac{3}{2} h^{2}\left[3 T^{3}-2 T-T^{-1}-4 T H\left(T^{2}-1+T H\right) T^{-1}\right], \\
C= & -3 h^{3}\left[\frac{15}{4}\left(T^{3}-T^{-3}\right)-\frac{9}{4}\left(T-T^{-1}\right)-\frac{9}{2} T H T-9 T H T^{-1}\right. \\
& \left.+\frac{23}{2} T H T^{-3}-9(T H)^{2}\left(T^{-3}-T^{-1}\right)+2(T H)^{3} T^{-3}\right], \\
D= & -2 h\left(T^{2}-1+2 T H\right) T^{-1}, \\
E= & -\frac{3}{2} h^{2}\left\{3\left(T+2 T^{-1}-3 T^{-3}\right)-4 T H\left[3\left(T^{2}-1\right)+T H\right] T^{-3}\right\}, \\
F= & -\frac{3}{2} h\left[3\left(T^{2}-1\right)+2 T H\right] T^{-3} .
\end{aligned}
$$

Upon applying the map (2.5), the matrices (2.17), (2.20), and (2.21), obtained using our contraction procedure, may be shown to be equal to the corresponding matrices (2.26)-(2.28), obtained directly using the expression (2.3) of the universal $\mathcal{R}_{h}$ matrix. This process may be continued indefinitely for arbitrary representations signifying that the arbitrary $h$-Jordanian matrices $R_{h}^{j_{1} ; j_{2}}$ may be recovered, via the contraction route (2.16), from the corresponding standard $R_{q}^{j_{1} ; j_{2}}$ matrices.

\section{On $\mathrm{U}_{h, \alpha}(\operatorname{gl}(2))$ and Its $R_{h, \alpha}$ Matrices}

We now consider the two-parametric $(h, \alpha)$-Jordanian deformed algebra $\mathrm{U}_{h, \alpha}(\operatorname{gl}(2)) \sim \mathrm{U}_{h, \alpha}(\operatorname{sl}(2) \oplus \mathrm{u}(1))$, generated by $(X, Y, H, Z)$ [8, 9]. The generator $Z$ of the $\mathrm{u}(1)$ algebra is a primitive central element. Our choice of the deformation parameters is a little different from others. Our results might be rewritten in terms of the customary choice of deformation parameters $h_{ \pm}=h(1 \pm \alpha)$. By studying the duality relation between the Hopf algebras $\operatorname{Fun}_{h, \alpha}(\mathrm{GL}(2))$ and $\mathrm{U}_{h, \alpha}(\operatorname{gl}(2))$, the Hopf structure of the latter has been investigated by Aneva et al. [9]. After a suitable choice of parameters, these authors conclude that the role of the second parameter may be confined to the coalgebra and the antipode alone.

Here we use the general formalism developed by Reshetikhin [23] for introducing multiple deformation parameters. This allows us to obtain the universal $\mathcal{R}_{h, \alpha}$ matrix 
of the two-parametric $\mathrm{U}_{h, \alpha}(\mathrm{gl}(2))$ algebra. To this end, we construct a twist operator

$$
F_{\alpha}=\exp [\alpha h(X \otimes Z-Z \otimes X)]
$$

Following Ref. [23], the coproducts of $\mathrm{U}_{h}(\mathrm{gl}(2))$ and $\mathrm{U}_{h, \alpha}(\operatorname{gl}(2))$ may be related by

$$
\Delta_{h, \alpha}(\mathcal{X})=F_{\alpha} \Delta_{h}(\mathcal{X}) F_{\alpha}^{-1} \quad \forall \mathcal{X} \in\{X, Y, H, Z\}
$$

Explicitly, we obtain

$$
\begin{aligned}
\Delta_{h, \alpha}(X)= & X \otimes 1+1 \otimes X \\
\Delta_{h, \alpha}(Y)= & Y \otimes T+T^{-1} \otimes Y+\alpha h\left(H \otimes Z T-Z T^{-1} \otimes H\right) \\
& -\frac{1}{2} \alpha^{2} h\left[Z^{2} T^{-1} \otimes\left(T-T^{-1}\right)+\left(T-T^{-1}\right) \otimes Z^{2} T\right], \\
\Delta_{h, \alpha}(H)= & H \otimes T+T^{-1} \otimes H+\alpha\left[Z T^{-1} \otimes\left(T-T^{-1}\right)-\left(T-T^{-1}\right) \otimes Z T\right], \\
\Delta_{h, \alpha}(Z)= & Z \otimes 1+1 \otimes Z .
\end{aligned}
$$

The antipode and the counit maps, however, remain unaltered. The authors of Ref. [9] obtained the antipode maps for $\mathrm{U}_{h, \alpha}(\mathrm{gl}(2))$ depending on both the deformation parameters. This result is, however, an artifact of their choice of the corresponding coproduct maps in a asymmetric way.

The universal $\mathcal{R}_{h, \alpha}$ matrix of the $\mathrm{U}_{h, \alpha}(\mathrm{gl}(2))$ algebra may also be determined following the Reshetikhin procedure 23:

$$
\mathcal{R}_{h, \alpha}=F_{\alpha}^{-1} \mathcal{R}_{h} F_{\alpha}^{-1}
$$

which, by construction, satisfies the properties (2.4), and the triangularity condition. Explicitly, $\mathcal{R}_{h, \alpha}$ reads

$$
\begin{aligned}
\mathcal{R}_{h, \alpha}= & \exp [-2 h \alpha(X \otimes Z-Z \otimes X)] \exp \left[-h\left(X \otimes T H+\alpha Z X \otimes\left(T^{2}-1\right)\right)\right] \\
& \times \exp \left[h\left(T H \otimes X-\alpha\left(T^{2}-1\right) \otimes Z X\right)\right] .
\end{aligned}
$$

The above universal $\mathcal{R}_{h, \alpha}$ matrix of the $(h, \alpha)$-Jordanian algebra $\mathrm{U}_{h, \alpha}(\operatorname{gl}(2))$ may also be obtained by the contraction technique discussed in Sect. 2. Our starting 
point here is the universal $\mathcal{R}_{q, \lambda}$ matrix [24] of the two-parametric $(q, \lambda)$-deformed standard $\mathrm{U}_{q, \lambda}(\mathrm{gl}(2))$ algebra [0]. Following Reshetikhin procedure [23], it was observed [24] that the universal $\mathcal{R}_{q, \lambda}$ matrix of the $\mathrm{U}_{q, \lambda}(\mathrm{gl}(2))$ algebra may be related to the universal $\mathcal{R}_{q}$ matrix of the standard $q$-deformed $\mathrm{U}_{q}(\operatorname{sl}(2))$ algebra via a twist operator:

$$
\mathcal{R}_{q, \lambda}=\hat{F}_{\lambda}^{-1} \mathcal{R}_{q} \hat{F}_{\lambda}^{-1}
$$

where $\hat{F}_{\lambda}$ is given by

$$
\hat{F}_{\lambda}=\lambda^{\frac{1}{2}\left(\hat{J}_{0} \otimes \hat{Z}-\hat{Z} \otimes \hat{J}_{0}\right)}=q^{\frac{\alpha}{2}\left(\hat{J}_{0} \otimes \hat{Z}-\hat{Z} \otimes \hat{J}_{0}\right)} .
$$

In the second equality in (3.7), we assume without any loss of generality $\lambda=q^{\alpha}$.

After taking into account the discussions in Sect. 2 about the convertibility, via the contraction process, of the standard $\mathcal{R}_{q}$ matrix of the $\mathrm{U}_{q}(\operatorname{sl}(2))$ algebra into the Jordanian $\mathcal{R}_{h}$ matrix of the $\mathrm{U}_{h}(\mathrm{sl}(2))$ algebra, a comparison between (3.4) and (3.6) makes it obvious that we need to show

$$
F_{\alpha}^{-1}=\lim _{q \rightarrow 1}\left(M^{-1} \otimes M^{-1}\right) \hat{F}_{\lambda}^{-1}(M \otimes M)
$$

Equation (3.8) may be readily proved as an operator identity. Using (3.7), and following the method developed in Ref. [18], we may show that the rhs of (3.8) assumes the form

$$
\begin{aligned}
\operatorname{rhs} & =\tilde{T}^{-\alpha z_{2}} \otimes \tilde{T}^{\alpha z_{1}} \\
& =\exp [-\alpha h(X \otimes Z-Z \otimes X)]=\text { lhs. }
\end{aligned}
$$

In the first equality in (3.9), the central generator $\hat{Z}$ of the $\mathrm{U}_{q, \lambda}(\operatorname{gl}(2))$ algebra is assumed to have values $z_{1}$ and $z_{2}$ in the two sectors of the tensor product space. In the second equality in (3.9), we have used the map (2.5), and the identity map $Z=\hat{Z}$. This completes our demonstration that the $(h, \alpha)$-Jordanian $R_{h, \alpha}^{j_{1}, z_{1} ; j_{2}, z_{2}}$ matrix of arbitrary coloured representation $\left(j_{1}, z_{1} \otimes j_{2}, z_{2}\right)$ may be recovered through the present contraction process from the corresponding two-parametric standard $R_{q, \lambda}^{j_{1}, z_{1} ; j_{2}, z_{2}}$ matrix. 
As an application of our method, we here obtain the matrices $R_{h, \alpha}^{\frac{1}{2}, z_{1} ; 1, z_{2}}$ and $R_{h, \alpha}^{1, z_{1} ; \frac{1}{2}, z_{2}}$, which satisfy appropriate coloured Yang-Baxter equation (11.1) with the central generator assuming different values in different sectors of the tensor product space:

$$
R_{h, \alpha}^{\frac{1}{2}, z_{1} ; 1, z_{2}}=\left(\begin{array}{cc}
A^{\prime} & B^{\prime} \\
0 & C^{\prime}
\end{array}\right), \quad R_{h, \alpha}^{1, z_{1} ; \frac{1}{2}, z_{2}}=\left(\begin{array}{ccc}
A^{\prime \prime} & B^{\prime \prime} & C^{\prime \prime} \\
0 & D^{\prime \prime} & E^{\prime \prime} \\
0 & 0 & F^{\prime \prime}
\end{array}\right)
$$

where $A^{\prime}, B^{\prime}, C^{\prime}$ are $3 \times 3$ matrices,

$$
\begin{aligned}
& A^{\prime}=\left(\begin{array}{ccc}
1 & 2 h\left(1+2 \alpha z_{1}\right) & 2 h^{2}\left(1+2 \alpha z_{1}\right)^{2} \\
0 & 1 & 2 h\left(1+2 \alpha z_{1}\right) \\
0 & 0 & 1
\end{array}\right), \\
& B^{\prime}=\left(\begin{array}{ccc}
-2 h\left(1+\alpha z_{2}\right) & 2 h^{2}\left(1-2 \alpha z_{1}\right. & 4 h^{3}\left(1+2 \alpha z_{1}-\alpha z_{2}\right. \\
0 & \left.-4 \alpha^{2} z_{1} z_{2}\right) & \left.-4 \alpha^{3} z_{1}^{2} z_{2}\right) \\
0 & -2 h \alpha z_{2} & 2 h^{2}\left(1+2 \alpha z_{1}-4 \alpha^{2} z_{1} z_{2}\right) \\
0 & 0 & 2 h\left(1-\alpha z_{2}\right)
\end{array}\right), \\
& C^{\prime}=\left(\begin{array}{ccc}
1 & -2 h\left(1-2 \alpha z_{1}\right) & 2 h^{2}\left(1-2 \alpha z_{1}\right)^{2} \\
0 & 1 & -2 h\left(1-2 \alpha z_{1}\right) \\
0 & 0 & 1
\end{array}\right)
\end{aligned}
$$

while $A^{\prime \prime}, B^{\prime \prime}, C^{\prime \prime}, D^{\prime \prime}, E^{\prime \prime}, F^{\prime \prime}$ are $2 \times 2$ matrices,

$$
\begin{aligned}
& A^{\prime \prime}=\left(\begin{array}{cc}
1 & 2 h\left(1+\alpha z_{1}\right) \\
0 & 1
\end{array}\right), \\
& B^{\prime \prime}=\left(\begin{array}{cc}
-2 h\left(1+2 \alpha z_{2}\right) & 2 h^{2}\left(1-2 \alpha z_{2}-4 \alpha^{2} z_{1} z_{2}\right) \\
0 & 2 h\left(1-2 \alpha z_{2}\right)
\end{array}\right), \\
& C^{\prime \prime}=\left(\begin{array}{cc}
2 h^{2}\left(1+2 \alpha z_{2}\right)^{2} & -4 h^{3}\left(1+2 \alpha z_{2}-\alpha z_{1}-4 \alpha^{3} z_{1} z_{2}^{2}\right) \\
0 & 2 h^{2}\left(1-2 \alpha z_{2}\right)^{2}
\end{array}\right), \\
& D^{\prime \prime}=\left(\begin{array}{cc}
1 & 2 h \alpha z_{1} \\
0 & 1
\end{array}\right), \\
& E^{\prime \prime}=\left(\begin{array}{cc}
-2 h\left(1+2 \alpha z_{2}\right) & 2 h^{2}\left(1+2 \alpha z_{2}-4 \alpha^{2} z_{1} z_{2}\right) \\
0 & 2 h\left(1-2 \alpha z_{2}\right)
\end{array}\right),
\end{aligned}
$$




$$
F^{\prime \prime}=\left(\begin{array}{cc}
1 & -2 h\left(1-\alpha z_{1}\right) \\
0 & 1
\end{array}\right) \text {. }
$$

Comparing the two matrices in (3.10), it is evident that an 'exchange symmetry' between the two sectors of the tensor product space holds:

$$
\left(R_{h, \alpha}^{j_{1}, z_{1} ; j_{2}, z_{2}}\right)_{k m, l n}=\left(R_{-h, \alpha}^{j_{2}, z_{2} ; j_{1}, z_{1}}\right)_{m k, n l} .
$$

\section{The Coloured $T_{h, \alpha}^{j, z}$ Matrices of the $\operatorname{Fun}_{h, \alpha}(\mathrm{GL}(2))$ Algebra}

In this section, we turn our attention to the determination of the $(h, \alpha)$-Jordanian $T$ matrices in arbitrary coloured representation. The contraction method described earlier may also be employed here. The technique may be summarized as follows. Starting with a two-parametric $(q, \lambda)$-standard $T_{q, \lambda}^{j, z}$ matrix of the $\operatorname{Fun}_{q, \lambda}(\mathrm{GL}(2))$ algebra in an arbitrary representation given by $j$, and the colour parameter $z$, we perform a suitable similarity transformation by the matrix $M_{j}$ defined in (2.15). Even though the transforming matrix is singular in the $q \rightarrow 1$ limit, the singularities all systematically cancel for the transformed matrix

$$
\tilde{T}^{j, z}=M_{j}^{-1} T_{q, \lambda\left(=q^{\alpha}\right)}^{j, z} M_{j},
$$

which yields finite elements of the corresponding two-parametric $(h, \alpha)$-Jordanian $T_{h, \alpha}^{j, z}$ matrix for the $(j, z)$ representation:

$$
T_{h, \alpha}^{j, z}=\lim _{q \rightarrow 1} \tilde{T}^{j, z} .
$$

The $T_{h, \alpha}^{j, z}$ matrix in (4.2) satisfies the coloured inverse scattering equation (1.2). For the fundamental representation $\left(j=\frac{1}{2}, z=\frac{1}{2}\right)$, the method was previously employed [13]. We first reproduce these results here for the notational purpose.

The generating elements $(\hat{a}, \hat{b}, \hat{c}, \hat{d})$ of the two-parametric standard $\operatorname{Fun}_{q, \lambda}(\mathrm{GL}(2))$ algebra, defined as $\hat{T}_{q, \lambda}^{\frac{1}{2}, \frac{1}{2}}=\left(\begin{array}{cc}\hat{a} & \hat{b} \\ \hat{c} & \hat{d}\end{array}\right)$, satisfy the following commutation relations

$$
\begin{aligned}
\hat{a} \hat{b} & =q^{-1} \lambda^{-1} \hat{b} \hat{a}, \quad \hat{a} \hat{c}=q^{-1} \lambda \hat{c} \hat{a}, \quad \hat{b} \hat{d}=q^{-1} \lambda \hat{d} \hat{b}, \quad \hat{c} \hat{d}=q^{-1} \lambda^{-1} \hat{d} \hat{c} \\
{[\hat{a}, \hat{d}] } & =\left(q^{-1}-q\right) \lambda^{-1} \hat{b} \hat{c}, \quad \lambda^{-1} \hat{b} \hat{c}=\lambda \hat{c} \hat{b}
\end{aligned}
$$


and the matrix coproduct rule $\Delta\left(\hat{T}_{q, \lambda}^{\frac{1}{2}, \frac{1}{2}}\right)=\hat{T}_{q, \lambda}^{\frac{1}{2}, \frac{1}{2}} \dot{\otimes} \hat{T}_{q, \lambda}^{\frac{1}{2}, \frac{1}{2}}$. The determinant $\hat{D}=$ $\hat{a} \hat{d}-q^{-1} \lambda^{-1} \hat{b} \hat{c}$ has the algebraic properties

$$
[\hat{a}, \hat{D}]=0, \quad \hat{b} \hat{D}=\lambda^{2} \hat{D} \hat{b}, \quad \hat{c} \hat{D}=\lambda^{-2} \hat{D} \hat{c}, \quad[\hat{d}, \hat{D}]=0
$$

and is endowed with a group-like coproduct $\Delta(\hat{D})=\hat{D} \otimes \hat{D}$.

The elements of the transformed $\tilde{T}^{\frac{1}{2}, \frac{1}{2}}=\left(\begin{array}{cc}\tilde{a} & \tilde{b} \\ \tilde{c} & \tilde{d}\end{array}\right)$ matrix, obtained following (4.1), are related to the old basis set as

$$
\hat{a}=\tilde{a}+\eta \tilde{c}, \quad \hat{b}=\tilde{b}-\eta(\tilde{a}-\tilde{d})-\eta^{2} \tilde{c}, \quad \hat{c}=\tilde{c}, \quad \hat{d}=\tilde{d}-\eta \tilde{c} .
$$

Staying away from the singularity in (4.5) in the $q \rightarrow 1$ limit, we recast the algebra (4.3) as

$$
\begin{aligned}
\tilde{a} \tilde{b}-q^{-1-\alpha} \tilde{b} \tilde{a} & =\eta\left(1-q^{-1-\alpha}\right)\left(\tilde{a}^{2}-\tilde{D}\right) \\
\tilde{a} \tilde{c}-q^{-1+\alpha} \tilde{c} \tilde{a} & =-\eta\left(1-q^{-1+\alpha}\right) \tilde{c}^{2} \\
\tilde{b} \tilde{d}-q^{-1+\alpha} \tilde{d} \tilde{b} & =-\eta\left(1-q^{-1+\alpha}\right)\left(\tilde{d}^{2}-\tilde{D}\right) \\
\tilde{c} \tilde{d}-q^{-1-\alpha} \tilde{d} \tilde{c} & =\eta\left(1-q^{-1-\alpha}\right) \tilde{c}^{2} \\
{[\tilde{a}, \tilde{d}] } & =\eta\left(1-q^{-1-\alpha}\right) \tilde{a} \tilde{c}-\eta\left(q^{1-\alpha}-1\right) \tilde{d} \tilde{c}+\left(q^{-1}-q\right) q^{-\alpha} \tilde{b} \tilde{c} \\
q^{-\alpha} \tilde{b} \tilde{c}-q^{\alpha} \tilde{c} \tilde{b} & =-\eta\left(q-q^{-\alpha}\right) \tilde{a} \tilde{c}-\eta\left(q-q^{\alpha}\right) \tilde{c} \tilde{d}
\end{aligned}
$$

where the determinant $\tilde{D}=\tilde{a} \tilde{d}-q^{-1-\alpha} \tilde{b} \tilde{c}-\eta\left(1-q^{-1-\alpha}\right) \tilde{a} \tilde{c}$ satisfies the commutation relations

$$
\begin{aligned}
{[\tilde{a}, \tilde{D}] } & =\eta\left(q^{2 \alpha}-1\right) \tilde{c} \tilde{D}, \quad \tilde{b} \tilde{D}-q^{2 \alpha} \tilde{D} \tilde{b}=\eta\left(q^{2 \alpha}-1\right)(\tilde{D} \tilde{d}-\tilde{a} \tilde{D}), \\
\tilde{c} \tilde{D}-q^{-2 \alpha} \tilde{D} \tilde{c} & =0, \quad[\tilde{d}, \tilde{D}]=-\eta\left(q^{2 \alpha}-1\right) \tilde{c} \tilde{D} .
\end{aligned}
$$

The elements obey the matrix coproduct rule $\Delta\left(\tilde{T}^{\frac{1}{2}, \frac{1}{2}}\right)=\tilde{T}^{\frac{1}{2}, \frac{1}{2}} \dot{\otimes} \tilde{T}^{\frac{1}{2}, \frac{1}{2}}$.

Defining the generating elements $(a, b, c, d)$ of the $\operatorname{Fun}_{h, \alpha}(\mathrm{GL}(2))$ algebra as $T_{h, \alpha}^{\frac{1}{2}, \frac{1}{2}}=\left(\begin{array}{ll}a & b \\ c & d\end{array}\right)$, we now pass to the $q \rightarrow 1$ limit in (4.6) and (4.7), and obtain the following:

$$
\begin{aligned}
& {[a, b]=h(1+\alpha)\left(a^{2}-D\right), \quad[a, c]=-h(1-\alpha) c^{2},} \\
& {[b, d]=-h(1-\alpha)\left(d^{2}-D\right), \quad[c, d]=h(1+\alpha) c^{2},} \\
& {[a, d]=h(1+\alpha) a c-h(1-\alpha) d c, \quad[b, c]=-h(1+\alpha) a c-h(1-\alpha) c d,(4.8)}
\end{aligned}
$$


where the determinant $D=a d-b c-h(1+\alpha) a c$ satisfies the commutation relations

$$
[a, D]=2 h \alpha c D, \quad[b, D]=2 h \alpha(D d-a D), \quad[c, D]=0, \quad[d, D]=-2 h \alpha c D
$$

The coproduct rules read

$$
\Delta\left(\begin{array}{ll}
a & b \\
c & d
\end{array}\right)=\left(\begin{array}{ll}
a & b \\
c & d
\end{array}\right) \dot{\otimes}\left(\begin{array}{ll}
a & b \\
c & d
\end{array}\right)
$$

It is interesting to observe that the algebraic relations (4.8) exhibit an automorphism

$$
\rho(a)=d, \quad \rho(b)=b, \quad \rho(c)=c, \quad \rho(d)=a,
$$

where the deformation parameters are also redefined according to $h \rightarrow h, \alpha \rightarrow-\alpha$. The map $\rho$ is also an anti-automorphism of the coalgebra rules (4.10):

$$
\sigma \circ(\rho \otimes \rho) \circ \Delta(\rho(\mathcal{X}))=\Delta(\mathcal{X}) \quad \forall \mathcal{X} \in\{a, b, c, d\}
$$

As the first example of our proposed generalization — as indicated in (4.1) and (4.2) - of the above construction method, we now consider $(h, \alpha)$-Jordanian $T_{h, \alpha}^{j=\frac{1}{2}, z}$ matrix for an arbitrary colour parameter $z$. The corresponding $(q, \lambda)$ standard $T_{q, \lambda}^{\frac{1}{2}, z}$ matrix for $\left(j=\frac{1}{2}, z\right)$ representation may be obtained [21] from the known expression [20] of the universal $\mathcal{T}_{q, \lambda}$ matrix acting as a dual form between the $\operatorname{Fun}_{q, \lambda}(\mathrm{GL}(2))$ and $\mathrm{U}_{q, \lambda}(\mathrm{gl}(2))$ algebras,

$$
\mathcal{T}_{q, \lambda}=\exp _{q^{2}}\left(\hat{\gamma} \hat{\mathcal{J}}_{-}\right) \exp \left[\hat{\alpha}\left(\frac{\hat{J}_{0}}{2}+\hat{Z}\right)+\hat{\delta}\left(\frac{\hat{J}_{0}}{2}-\hat{Z}\right)\right] \exp _{q^{-2}}\left(\hat{\beta} \hat{\mathcal{J}}_{+}\right)
$$

where $\hat{\mathcal{J}}_{+}=\hat{J}_{+} q^{\left(\hat{J}_{0}+1\right) / 2} \lambda^{\hat{Z}-\frac{1}{2}}$ and $\hat{\mathcal{J}}_{-}=q^{-\left(\hat{J}_{0}+1\right) / 2} \lambda^{\hat{Z}-\frac{1}{2}} \hat{J}_{-}$. The redefined generating elements of the $\operatorname{Fun}_{q, \lambda}(\mathrm{GL}(2))$ algebra are given by

$$
\hat{a}=e^{\hat{\alpha}}, \quad \hat{b}=e^{\hat{\alpha}} \hat{\beta}, \quad \hat{c}=\hat{\gamma} e^{\hat{\alpha}}, \quad \hat{d}=\hat{\gamma} e^{\hat{\alpha}} \hat{\beta}+e^{-\hat{\delta}}
$$

For the $\left(j=\frac{1}{2}, z\right)$ representation, the $(q, \lambda)$-deformed standard $\hat{T}_{q, \lambda}^{\frac{1}{2}, z}$ matrix reads 21]

$$
\hat{T}_{q, \lambda}^{\frac{1}{2}, z}=\hat{D}^{z-\frac{1}{2}}\left(\begin{array}{cc}
\hat{a} & \lambda^{z-\frac{1}{2} \hat{b}} \\
\lambda^{-\left(z-\frac{1}{2}\right)} \hat{c} & \hat{d}
\end{array}\right)
$$


Using the transformation (4.1) and the redefinition of the variables (4.5), we now obtain

$$
\tilde{T}^{\frac{1}{2}, z}=M_{\frac{1}{2}}^{-1} \hat{T}_{q, \lambda\left(=q^{\alpha}\right)}^{\frac{1}{2}, z} M_{\frac{1}{2}}
$$

as

$$
\tilde{T}^{\frac{1}{2}, z}=\hat{D}^{z-\frac{1}{2}}\left(\begin{array}{cc}
\tilde{a}+\eta\left(1-q^{-\alpha\left(z-\frac{1}{2}\right)}\right) \tilde{c} & q^{\alpha\left(z-\frac{1}{2}\right)} \hat{b}-\eta\left(q^{\alpha\left(z-\frac{1}{2}\right)}-1\right)(\tilde{a}-\tilde{d}) \\
q^{-\alpha\left(z-\frac{1}{2}\right)} \tilde{c} & -\eta^{2}\left(q^{\alpha\left(z-\frac{1}{2}\right)}+q^{-\alpha\left(z-\frac{1}{2}\right)}-2\right) \tilde{c} \\
\tilde{d}-\eta\left(1-q^{-\alpha\left(z-\frac{1}{2}\right)}\right) \tilde{c}
\end{array}\right) .
$$

The limiting process $q \rightarrow 1$ in 4.17 now yields the desired $(h, \alpha)$-Jordanian $T_{h, \alpha}^{\frac{1}{2}, z}$ matrix for the coloured $\left(j=\frac{1}{2}, z\right)$ representation,

$$
T_{h, \alpha}^{\frac{1}{2}, z}=D^{z-\frac{1}{2}}\left(\begin{array}{cc}
a+h \alpha\left(z-\frac{1}{2}\right) c & b-h \alpha\left(z-\frac{1}{2}\right)(a-d)-h^{2} \alpha^{2}\left(z-\frac{1}{2}\right)^{2} c \\
c & d-h \alpha\left(z-\frac{1}{2}\right) c
\end{array}\right)
$$

which satisfies the appropriate coloured inverse scattering equation (1.2). Substitution of the $z=\frac{1}{2}$ value of the colour parameter obviously reduces (4.18) to the fundamental representation.

As another demonstration of our procedure, we explicitly obtain the $(h, \alpha)$ Jordanian $T_{h, \alpha}^{1, z}$ matrix for $j=1$ representation at an arbitrary value of the colour parameter $z$. As previously, our starting point here is the $(q, \lambda)$-standard $\hat{T}_{q, \lambda}^{1, z}$ matrix of the $(1, z)$ representation. Using the representation (2.18) and the universal $\mathcal{T}_{q, \lambda}$ matrix (4.13), we obtain

$$
\hat{T}_{q, \lambda}^{1, z}=\hat{D}^{z-1}\left(\begin{array}{ccc}
\hat{a}^{2} & {[2]_{q} q^{\frac{1}{2}} \lambda^{z-\frac{1}{2}} \hat{a} \hat{b}} & {[2]_{q} \lambda^{2(z-1)} \hat{b}^{2}} \\
q^{\frac{1}{2}} \lambda^{-z+\frac{1}{2}} \hat{a} \hat{c} & \hat{a} \hat{d}+q \lambda^{-1} \hat{b} \hat{c} & {[2]_{q} q^{\frac{1}{2}} \lambda^{z-\frac{3}{2}} \hat{b} \hat{d}} \\
{[2]_{q}^{-1} \lambda^{-2(z-1)} \hat{c}^{2}} & q^{\frac{1}{2}} \lambda^{-z+\frac{3}{2}} \hat{c} \hat{d} & \hat{d}^{2}
\end{array}\right)
$$

Using the construction of the transforming matrix $M_{j=1}$ in (2.19), we first perform the similarity transformation (4.1), and using (4.5) reexpress the elements of the relevant $\tilde{T}^{1, z}$ matrix in terms of the variables $(\tilde{a}, \tilde{b}, \tilde{c}, \tilde{d})$. The transformed matrix is now free from singularities at all orders as $q \rightarrow 1$. Consequently, in the said 
$q \rightarrow 1$ limit, we now explicitly obtain finite elements of the corresponding $(h, \alpha)$ Jordanian $T_{h, \alpha}^{1, z}$ matrix for the $(j=1, z)$ representation,

$$
T_{h, \alpha}^{1, z}=D^{z-1}\left(\begin{array}{ccc}
U_{11}^{z} & U_{12}^{z} & U_{13}^{z} \\
U_{21}^{z} & U_{22}^{z} & U_{23}^{z} \\
U_{31}^{z} & U_{32}^{z} & U_{33}^{z}
\end{array}\right) \text {, }
$$

where

$$
\begin{aligned}
& U_{11}^{z}=a^{2}+2 h \alpha(z-1) a c+\frac{1}{4} h^{2}[1+\alpha(2 z-3)]^{2} c^{2}, \\
& U_{12}^{z}=2 a b-h[1+\alpha(2 z-1)]\left(a^{2}-a d\right)-h[1-\alpha(2 z-3)] b c \\
& -h^{2}\left[1+2 \alpha+\alpha^{2}\left(4 z^{2}-8 z+5\right)\right] a c+\frac{1}{2} h^{2}[1+\alpha(2 z-3)]^{2} c d \\
& -\frac{1}{4} h^{3}\left[1+\alpha(6 z-7)+\alpha^{2}\left(12 z^{2}-28 z+15\right)\right. \\
& \left.+\alpha^{3}\left(8 z^{3}-28 z^{2}+30 z-9\right)\right] c^{2}, \\
& U_{13}^{z}=2 b^{2}-4 h \alpha(z-1)(a b-b d)-\frac{1}{2} h^{2}\left[3-2 \alpha(2 z-1)-\alpha^{2}(2 z-1)^{2}\right] a^{2} \\
& +h^{2}\left[1-4 \alpha(z-1)-\alpha^{2}\left(4 z^{2}-8 z+5\right)\right] a d \\
& -h^{2}\left[1-4 \alpha(z-1)+\alpha^{2}\left(4 z^{2}-8 z+3\right)\right] b c+\frac{1}{2} h^{2}[1+\alpha(2 z-3)]^{2} d^{2} \\
& -h^{3}\left[1-\alpha(z-2)-\alpha^{2}(6 z-5)-\alpha^{3}\left(4 z^{3}-12 z^{2}+13 z-4\right)\right] a c \\
& -h^{3}\left[\alpha(z-1)+2 \alpha^{2}\left(2 z^{2}-5 z+3\right)+\alpha^{3}\left(4 z^{3}-16 z^{2}+21 z-9\right)\right] c d \\
& -\frac{1}{8} h^{4}\left[3+8 \alpha(z-2)-2 \alpha^{2}\left(4 z^{2}-7\right)-8 \alpha^{3}\left(4 z^{3}-12 z^{2}+11 z-3\right)\right. \\
& \left.-\alpha^{4}\left(16 z^{4}-64 z^{3}+88 z^{2}-48 z+9\right)\right] c^{2}, \\
& U_{21}^{z}=a c+\frac{1}{2} h[1+\alpha(2 z-3)] c^{2}, \\
& U_{22}^{z}=a d+b c-2 h \alpha(z-1) a c+h[1+\alpha(2 z-3)] c d \\
& -\frac{1}{2} h^{2}\left[1+4 \alpha(z-1)+\alpha^{2}\left(4 z^{2}-8 z+3\right)\right] c^{2}, \\
& U_{23}^{z}=2 b d+h[1+\alpha(2 z-3)]\left(d^{2}-a d\right)+h[1-\alpha(2 z-1)] b c \\
& -\frac{1}{2} h^{2}\left[1-2 \alpha-\alpha^{2}\left(4 z^{2}-8 z+3\right)\right] a c-2 h^{2} \alpha[z-1 \\
& \left.+\alpha\left(2 z^{2}-5 z+3\right)\right] c d-\frac{1}{4} h^{3}[3+\alpha(2 z-7) \\
& \left.-\alpha^{2}\left(12 z^{2}-20 z+7\right)-\alpha^{3}\left(8 z^{3}-20 z^{2}+14 z-3\right)\right] c^{2}, \\
& U_{31}^{z}=\frac{1}{2} c^{2}
\end{aligned}
$$




$$
\begin{aligned}
& U_{32}^{z}=c d-\frac{1}{2} h[1+\alpha(2 z-1)] c^{2} \\
& U_{33}^{z}=d^{2}-2 h \alpha(z-1) c d-\frac{1}{4} h^{2}\left[3-2 \alpha(2 z-1)-\alpha^{2}(2 z-1)^{2}\right] c^{2} .
\end{aligned}
$$

For the usual choice of the colour parameter $z=\frac{1}{2}$, the $T_{h, \alpha}^{j=1, z=\frac{1}{2}}$ matrix is obtained from (4.20) and (4.21):

$$
T_{h, \alpha}^{1, \frac{1}{2}}=D^{-1 / 2}\left(\begin{array}{ccc}
U_{11} & U_{12} & U_{13} \\
U_{21} & U_{22} & U_{23} \\
U_{31} & U_{32} & U_{33}
\end{array}\right)
$$

where

$$
\begin{aligned}
U_{11}= & a^{2}-h \alpha a c+\frac{1}{4} h^{2}(1-2 \alpha)^{2} c^{2} \\
U_{12}= & 2 a b-h\left(a^{2}-a d\right)-h(1+2 \alpha) b c-h^{2}\left(1+2 \alpha+2 \alpha^{2}\right) a c \\
& +\frac{1}{2} h^{2}(1-2 \alpha)^{2} c d-\frac{1}{4} h^{3}(1-2 \alpha)^{2} c^{2} \\
U_{13}= & 2 b^{2}+2 h \alpha(a b-b d)-\frac{3}{2} h^{2} a^{2}+h^{2}\left(1+2 \alpha-2 \alpha^{2}\right) a d \\
& -h^{2}(1+2 \alpha) b c+\frac{1}{2} h^{2}(1-2 \alpha)^{2} d^{2}-\frac{1}{2} h^{3}\left(2+3 \alpha+4 \alpha^{2}\right) a c \\
& +\frac{1}{2} h^{3} \alpha(1-2 \alpha)^{2} c d-\frac{3}{8} h^{4}(1-2 \alpha)^{2} c^{2}, \\
U_{21}= & a c+\frac{1}{2} h(1-2 \alpha) c^{2}, \\
U_{22}= & a d+b c+h \alpha a c+h(1-2 \alpha) c d-\frac{1}{2} h^{2}(1-2 \alpha) c^{2}, \\
U_{23}= & 2 b d+h(1-2 \alpha)\left(d^{2}-a d\right)+h b c-\frac{1}{2} h^{2}(1-2 \alpha) a c \\
& +h^{2} \alpha(1-2 \alpha) c d-\frac{3}{4} h^{3}(1-2 \alpha) c^{2}, \\
U_{31}= & \frac{1}{2} c^{2}, \\
U_{32}= & c d-\frac{1}{2} h c^{2}, \\
U_{33}= & d^{2}+h \alpha c d-\frac{3}{4} h^{2} c^{2} .
\end{aligned}
$$

The elements of $T_{h, \alpha}^{1, \frac{1}{2}}$ in (4.22) and (4.23) may be rewritten in terms of the alternative choice of deformation parameters $h_{ \pm}$.

The $T_{h}^{1, \frac{1}{2}}$ matrix, corresponding to a single Jordanian deformation parameter $h$, 
may be obtained by choosing $\alpha=0$ (i.e. $h_{+}=h_{-}=h$ ) in (4.22) and (4.23):

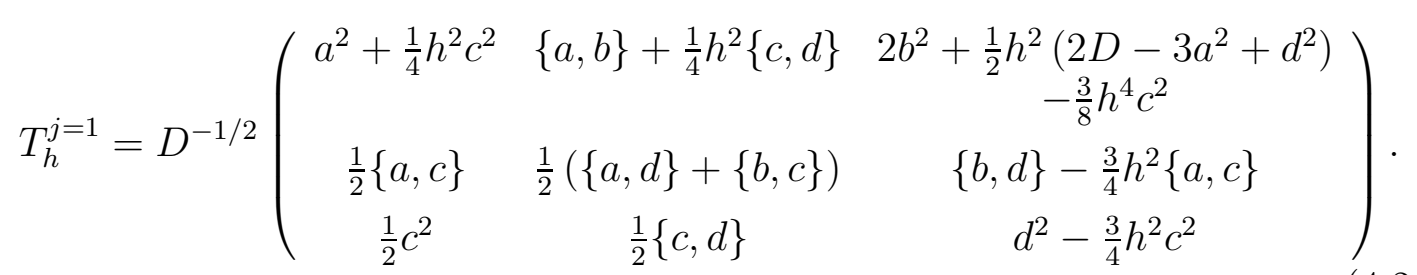

Using the commutation relations, obtained from (4.8), corresponding to the singleparametric case $(\alpha=0)$, we have expressed the elements of the $T_{h}^{j=1}$ matrix in (4.24) in terms of the determinant, and the anticommutators of the generating elements.

The contraction technique, comprising of the processes (4.1) and (4.2), may be continued for obtaining the $(h, \alpha)$-Jordanian monodromy matrices $T_{h, \alpha}^{j, z}$ for arbitrary coloured $(j, z)$ representations. Singularities systematically cancel leading to a welldefined method of evaluation of the higher-dimensional $T_{h, \alpha}^{j, z}$ matrices.

\section{Conclusion}

Extending earlier results, we have shown that the $h$-deformed Jordanian $R_{h}^{j_{1} ; j_{2}}$ matrices may be obtained from the corresponding standard $q$-deformed $R_{q}^{j_{1} ; j_{2}}$ matrices through the present contraction process. In this demonstration, a key role is played by a nonlinear map of the $\mathrm{U}_{h}(\mathrm{sl}(2))$ algebra on the classical $\mathrm{U}(\mathrm{sl}(2))$ algebra. The Drinfeld twist operator relating the $h$-Jordanian and the classical coproduct structures may be determined as a series expansion in $h$ up to an arbitrary order. The twist operator interrelating the corresponding antipode maps has been determined in a closed form. By using the Reshetikhin formalism, the universal $\mathcal{R}_{h, \alpha}$ matrix of the two-parametric $(h, \alpha)$ Jordanian deformation has been obtained.

Moreover, we have shown that the two-parametric $(q, \lambda)$ standard $R_{q, \lambda}^{j_{1}, z_{1} ; j_{2}, z_{2}}$ matrices of arbitrary coloured $\left(j_{1}, z_{1} \otimes j_{2}, z_{2}\right)$ representations reduce - via the contraction procedure - to the corresponding $(h, \alpha)$-Jordanian coloured matrices.

Finally, we have proved that the contraction process may serve as a useful tool to explicitly extract the so far unknown $(h, \alpha)$-Jordanian $T_{h, \alpha}^{j, z}$ matrices for arbitrary 
$(j, z)$ coloured representations from the corresponding $(q, \lambda)$-deformed standard $T_{q, \lambda}^{j, z}$ matrices.

Our method may be useful in formulating Jordanian $h$-special functions in analogy with the standard $q$-special functions. Of special relevance is the well-known result [25] that the elements of the standard $T_{q}^{j}$ matrices may be explicitly expressed in terms of the little $q$-Jacobi polynomials. It would be of interest to explore if a Jordanian analogue of this result holds. We hope to address this question elsewhere.

\section{Acknowledgments}

We thank A. Chakrabarti, R. Jagannathan, and J. Segar for useful discussions. One of us (RC) wishes to thank C. Quesne for a kind invitation. He is also grateful to the members of the PNTPM group for their kind hospitality. 


\section{References}

[1] B. A. Kupershmidt, J. Phys. A25, L1239 (1992).

[2] V. G. Drinfeld, in Proc. Int. Congress of Mathematicans (Berkeley, CA, 1986), ed. A. M. Gleason (AMS, Providence, RI, 1987), p. 798.

[3] E. E. Demidov, Yu. I. Manin, E. E. Mukhin and D. V. Zhdanovich, Prog. Theor. Phys. Suppl. 102, 203 (1990); S. Zakrzewski, Lett. Math. Phys. 22, 287 (1991).

[4] Ch. Ohn, Lett. Math. Phys. 25, 85 (1992).

[5] A. Ballesteros and F. J. Herranz, J. Phys. A29, L311 (1996); A. Shariati, A. Aghamohammadi and M. Khorrami, Mod Phys. Lett. A11, 187 (1996).

[6] V. K. Dobrev, in Proc. of the 10th Int. Conf. 'Problems of Quantum Field Theory', (Alushta, Crimea, Ukraine, 13-18.5.1996), eds. D. Shirkov, D. Kazakov and A. Vladimirov, JINR E2-96-369 (Dubna, 1996), p. 104.

[7] A. Schirrmacher, J. Wess and B. Zumino, Z. Phys. C49, 317 (1991).

[8] A. Aghamohammadi, Mod. Phys. Lett. A8, 2607 (1993).

[9] B. L. Aneva, V. K. Dobrev and S. G. Milov, J. Phys. A30, 6769 (1997).

[10] P. Parashar, "Jordanian $\mathrm{U}_{h, s} \mathrm{gl}(2)$ and its coloured realization," preprint $\mathrm{q}$ alg/9705027, Lett. Math. Phys. (in press).

[11] C. Quesne, J. Math. Phys. 38, 6018 (1997).

[12] B. Basu-Mallick, Int. J. Mod. Phys. A10, 2851 (1995).

[13] A. Aghamohammadi, M. Khorrami and A. Shariati, J. Phys. A28, L225 (1995), " $h$-Deformation as a contraction of $q$-deformation," preprint hep-th/9410135.

[14] M. Alishahiha, J. Phys. A28, 6187 (1995). 
[15] B. Abdesselam, A. Chakrabarti and R. Chakrabarti, Mod. Phys. Lett. A11, 2883 (1996).

[16] N. Aizawa, J. Phys. A30, 5981 (1997), "Tensor operators for $\mathrm{U}_{h}(\mathrm{sl}(2))$," preprint math.QA/9803147, Lett. Math. Phys. (in press).

[17] J. Van der Jeugt, J. Phys. A31, 1495 (1998).

[18] B. Abdesselam, A. Chakrabarti and R. Chakrabarti, Mod. Phys. Lett. A13, 779 (1998).

[19] V. G. Drinfeld, Leningrad Math. J. 1, 1419 (1990).

[20] C. Fronsdal and A. Galindo, Lett. Math. Phys. 27, 59 (1993).

[21] R. Jagannathan and J. Van der Jeugt, J. Phys. A28, 2819 (1995).

[22] S. Majid, Foundations of Quantum Group Theory (Cambridge University Press, Cambridge, 1995).

[23] N. Reshetikhin, Lett. Math. Phys. 20, 331 (1990).

[24] R. Chakrabarti and R. Jagannathan, J. Phys. A27, 2023 (1994).

[25] L. L. Vaksman and Ya. S. Soibel'man, Func. Anal. Appl. 22, 170 (1988). 